tion. They attributed this inhibition to Haemaccel, a plasma expander used in the heart-lung machine. We have found, however, that heparin also strongly inhibits ristocetin-induced platelet aggregation.

In our in-vitro experiments by the turbidimetrical method using heparin PRP (250 $\mathrm{U} / 1)$ aggregation due to ristocetin in a concentration of $1 \mathrm{~g} / 1$ was completely abolished by this substance, while with $1.5 \mathrm{~g}$ ristocetin/1 the inhibitory effect of heparin started at a concentration of $10 \mathrm{kU} / 1$ and was completed at $25 \mathrm{kU} / 1$.

In blood obtained from 10 normal individuals after an intravenous injection of $5000 \mathrm{U}$ of heparin there was a slight impairment of aggregation with ristocetin $(1.5 \mathrm{~g} / 1)$, while with $1 \mathrm{~g}$ ristocetin/1 platelet aggregation was abnormal.

We conclude therefore that heparin as well as Haemaccel must play a part in the inhibition of ristocetin-induced platelet aggregation in patients undergoing cardiopulmonary bypass.

Department of Haematology,

Clinic of Internal Medicine,

Çapa, Istanbul, Turkey

\section{Serum digoxin in patients with thyroid disease}

SIR,-The paper by Dr M S Croxson and Professor H K Ibbertson (6 September, p 566) was an interesting study of drug metabolism in thyroid disease.

The low serum digoxin levels in thyrotoxicosis are probably due in part to increased clearance of the drug, as suggested. But a much more important factor may be that of malabsorption, which was not adequately investigated.

It is known that a high percentage of patients with thyrotoxicosis have steatorrhoea. ${ }^{1}$ Digoxin is a steroid molecule and poorly soluble in water. It is therefore very probable that malabsorption of digoxin accompanies the steatorrhoea of thyrotoxicosis. Unfortunately $\mathrm{Dr}$ Croxson and Professor Ibbertson measured digoxin excretion in only two patients with thyrotoxicosis and no control values were given, so we feel that there is little evidence to support their suggestion that malabsorption does not play an important role in the low serum digoxin levels in thyrotoxicosis. This aspect obviously needs further investigation, especially as the results may have implications for other drugs given to patients with thyrotoxicosis.

KENNETH WATTERS G H TOMKIN

Metabolic Unit,

Adelaide Hospital,

Dublin

1 Thomas, F B, Caldwell, J $\mathrm{H}$, and Greenberger,

\section{Rabies}

SIR,-In your leading article on the diagnosis and treatment of human rabies (27 September, $p$ 721) you do not include the valuable evidence that can of ten be obtained about the animal. The state of the dog, for example, is very relevant in making a correct diagnosis. When I worked in Zambia I learnt to ask all about the "mad" dog. If it is rabid it dies within 10 days. I checked this fact with the Liverpool School of Tropical Medicine.

When faced with a patient showing symptoms and signs suggestive of rabies there may be clear-cut evidence that the dog was ill and died of its illness. Rabies becomes probable. If the history is of no dog bite, or a bite by a dog that was not ill and that was known to be alive 10 days afterwards, rabies is nevertheless still a possibility because of the unreliability of negative evidence.

But the question whether there has been inoculation of the virus often arises when someone is bitten or has contact with an ill dog. Here the state of the animal is of essential importance. If alive it must be compounded and kept to see if it dies within 10 days. All too often a dog is killed in anger, ignorance, or misplaced zeal, although there may be circumstances when it is too mad to be caught and kept alive. It must not be destroyed or buried. A post-mortem examination is obligatory, and of course this is most likely to give reliable results if the dog has died naturally. If it cannot be proved that the dog was free from rabies, then prophylactic injections must be given to the patient and others at risk, even though they are of ten painful and hazardous. I raise this as only last week a patient asked about a bite from a dog "from Germany." The dog was alive and well and had been in quarantine, so I was able to reassure them that there was no danger of rabies.

Kirkham

J E PARRY

SIR,-Your timely leading article on the diagnosis and management of human rabies (27 September, p 721) was extremely interesting. However, I believe it would also be very desirable to hear from one of our veterinary colleagues on public health aspects of the disease in animals in view of the spread of rabies across Europe.

Many people think of animal rabies only in terms of the furious form of the disease, whereas in the tropical countries in which I have served I think it was the animal with the dumb form which was more dangerous because the state of the animal could not be immediately recognised.

H B L RUSSELI

University Department of Community Medicine, Edinburgh

\section{Controlled trial of therapy in Reye's syndrome}

SIR,-Your timely leading article on Reye's syndrome (20 September, p 662) correctly emphasises the importance of considering this diagnosis in any child with convulsions and coma. Since the pathophysiology of this condition is so poorly understood the value of the various forms of therapy which have been advocated for this condition can be decided only by well-conducted controlled trials. A major problem in instituting such trials is the comparative rarity of the condition, its sporadic frequency, and its varying severity. None of the forms of therapy mentioned in the leading article have been subjected to such controlled trials. For that reason we are co-operating in a multicentre controlled trial organised by the department of paediatrics of Yale University in conjunction with some eight units.

Four treatment regimens are being assessed, supportive treatment as outlined in your leading article being compared with similar supportive measures aided by (a) exchange transfusion, (b) peritoneal dialysis, and (c) glucose and insulin infusion.

We would be pleased to supply further details of the trial protocol to clinicians who would wish to participate in this trial. May we through your columns also ask any pathologists or clinicians to preserve serum, urine, and liver tissue from such patients, preferably at a temperature of $-70^{\circ} \mathrm{C}$, so that these may be available for analysis as the pathophysiology of this condition is elucidated?

King's College Hospital,

ALEX P MOWAT

London SE5

Guy's Hospital,

B G R NEVILLE

SE1

\section{SI units}

SIR,-Among doctors divorced from the hierarchy of the teaching hospitals and pure science there is an overwhelming feeling that we are being conned into accepting the introduction of SI units as an advancement in medical technique. One district after another in Kent through their district medical committees have already sent resolutions of protest and at least one district pharmaceutical committee and the area medical advisory committee have similarly protested.

There are no reasons to suppose that the introduction of SI units will benefit the patient or improve the results of investigations interpreted through SI units; indeed, the reverse is likely to occur, with possible disastrous results to the patient. The undue haste with which this scheme is being introduced will bring intolerable pressures on both the medical and nursing professions, and it seems highly unlikely that reeducation of such large numbers can be completed in time. That such a scheme should be introduced at this time of financial stringency and shortage of all grades of hospital staff to meet the whims of scientific bureaucrats and none else seems quite incredible. We readily support our colleagues on these other committees and ask that in the interests of the patient and the country the introduction of SI units shall be postponed, possibly for even as long as five years.

A F CRICK Honorary Secretary

Dartford. Gravesend, and Medway Division, BMA

Graverend, Kent

\section{Effects of exertion on hormone secretion}

SIR,-In addition to the investigations presented in our previous letters (29 June 1974, p 726; 21 June 1975, p 685) we decided to assay plasma growth hormone (GH) before and after exertion in the remaining sera from the group of amateur Finnish marathon runners. The determination of $\mathrm{GH}$ was performed with a double-antibody solid-phase technique. Mean control values were 0.7 (range $0 \cdot 2-2 \cdot 8) \mu \mathrm{g} / 1$ and after the run the mean value was 5.4 (range 1.3-11.2) $\mu \mathrm{g} / 1$ 